\title{
On Evolution and Development Frontier of 3D Printing Technology Based on Document Analysis
}

\author{
Guodong Tian \\ Nanjing Agricultural University, Nanjing Jiangsu, 210000, China
}

Key words: Document analysis, 3D printing technology, Evolution, Development frontier, Research.

\begin{abstract}
Under the new period, the emergence of 3D printing technology changes the world, so it receives much attention of various fields. The cultivation of 3D printing technology plays an irreplaceable role in transforming and upgrading national high-end manufacturing industry as well as enhancing core competitiveness. On this basis, this paper deeply discusses the research hotspot of 3D printing technology field with bibliometrics and multiple kinds of software. Besides, this paper describes in detail the development situation of 3D printing technology, expounds four mainstream research fields, explores the evolution and development course of this technology and gains the mapping knowledge domain about the evolution and research frontier of 3D printing technology in future 5 years. Finally, corresponding conclusions are obtained, in the hope of laying a solid foundation for further development of 3D printing technology.
\end{abstract}

\section{Research overview of 3D printing technology}

In current stage, 3D printing technology has become a key technology which changes the world. Thus, it receives much attention globally. The Economist clearly indicates that this technology along with other digital production mode will drive the third industrial revolution. In the development process of this technology, numerous subversive innovations also occur. 3D printing technology will enter the practical application stage.

Under the background of post-economic crisis, various countries are looking for the latest economic growth point. Meanwhile, they will continue to cultivate more competitive new technologies $^{[1]}$. 3D printing technology is the most representative technology, and will play a crucial role in economic development. Many countries also regard this technology as the key part of manufacturing industry and other industries and carry out further deployment and planning.

It thus can be seen that the function of 3D printing technology gradually stands out in the manufacturing field. In current stage, domestic and overseas scholars have implemented deeply researches on relevant fields from technical perspective. However, the analyses from the perspective of patent and document are few. Dong Kun and Liu Hongguang et al. preliminarily studied 3D printing technology from patent or document perspective. As well, many foreign research institutions also researched this technology from such perspective, but few scholars deeply discuss the evolution process and research frontier of 3D printing technology. Although some scholars analyzed 3D printing technology from the patent perspective, many researches and analyses focus on multiple processes of 3D printing technology, because most patents concentrate on manufacturing improvement. However, for most countries, 3D printing technology is not just technical cultivation. They deeply study industrialization development of 3D printing.

\section{Data acquisition and research method analysis}

Bibliometrics quantification refers to detection and confirmation of production and development of new technology. It is a technology with fundamentality and effectiveness. However, effectiveness of 
bibliometrics analysis must be based on document data collection. In practical analysis process, most scholars will usually apply SCI and reflect the academic value of documents. Meanwhile, they can effectively construct network system of references in the research field. Hence, this paper chooses SSCI and SCI-E databases in WoS, because SSCI and SCI-E databases include important journals about 3D printing technology.

In this research, original documents and searching vocabulary about 3D printing will be summarized. Besides, the technology related to 3D printing will be added to guarantee the integrity degree of documents searched. Since 3D printing field starts to develop in 1980s, the years of document retrieval was set to be $1985-2014^{[2]}$. For the purpose of further analysis, documents and records were collected in an all-round way, mainly including document titles, abstracts, authors, expended key words and documents cited etc. The number of documents collected exceeded 7431. Data cleaning and repetition removal were carried out for all documents collected. Finally, 7368 documents were left to make sure the documents were more accurate. The analysis tools include Bibexcel document analysis software, Citespace software and HistCite software.

\section{Analysis and elaboration of research results}

\section{Research status}

Rational application and analysis of Citespace 3 can offer effective research basis for the development of 3D printing technology. The software takes co-citation analysis network and co-word network as the important foundation and belongs to measuring software of knowledge visualization. The software can automatically eliminate the data information unrelated to the research theme. Thus, it has certain applicability in co-citation analysis.

The documents collected were inputted in the software, and the valid time was set to be 3 years. The articles ranking top 50 in terms of citation quantity were chosen at each interval. Path search algorithm was applied to carry out co-citation analysis of research documents about 3D printing technology and gain cluster mapping knowledge domain related to co-citation analysis and 3D printing.

Through the research on the mapping knowledge domain, we can find that the cited documents as the important basis form key words of the research field. The key words are gathered to form the specific research area. For this research field, documents of some scholars can gain this group research field. The data set can be divided according to the clustering result, and 39 groups form. Typical academic groups cover multiple concepts such as rapid prototyping, mechanical process performance and 3D model technology ${ }^{[3]}$. At present, frontier research field is still an emerging research field or the non-mainstream research field. Thus, the data information about the scholars is gained from this paper and the articles ranking top 10 in terms of citation quantity in the research field.

According to information summary and research, we can know the research on 3D printing technology mainly includes two aspects: firstly, further improve the technology involved in the manufacturing process; secondly, fully consider the features of application field, and carry out all-round and deep researches on improvement of material and bioengineering technology.

On this basis, correlation analysis should be carried out for the research field of 3D printing. The author co-citation regards document author as the basic unit to form co-citation relationship. The application of this relation can make the authors whose documents are cited concentrate in the uniform subject group to construct subject group network. In the process of reflecting the structure feature of this field, the relation among the cited authors and development situation can be truly reflected. In such case, author co-citation and information visualization may be used to further study and discuss academic field development of 3D printing technology.

For the articles downloaded, the scholars with the citation quantity exceeding 50 were chosen. After the name processing, 38 scholars were chosen, and the total frequency of occurrence was 3938. Besides, the occurrence frequency of every author exceeded 100. Then, Bibexcel software was 
applied. Meanwhile, author co-citation analysis was made to get the co-citation matrix of 38 scholars. Then, SPSS software was used to transform the matrix and deeply analysis the principal component. Through multi-dimensional and multi-scale analysis, clustering analysis results were combined to draw mapping knowledge domain of 3D printing technology research. In this way, the authors with high frequency could be shown vividly. In addition, the research fields and themes of highly-cited authors could be exhibited.

Based on applying the results of principal component analysis, searching and sorting the documents published by different authors, the concentrated field of authors and research themes about 3D printing field were gained. Since the types of periodicals and documents in each research field are different, the development features of 3D printing technology are also proven, i.e. wide types of industries covered, and obvious intersection of subjects ${ }^{[4]}$.

\section{Research frontier and future development trend}

Some scholars think that the terminologies with fast frequency increase speed can be chosen in the documents to test the growth of the research interest. Generally speaking, the application of citation explosiveness can carried out the research on an indicator of the most active part in a field. Citation explosiveness can test the events keeping long-term citation explosion, often called the hotspot in the research field. Through screening the documents for 10 years, 10 documents with the strongest citation explosiveness are summarized. It is found from tracking analysis that the research hotspot in recent years is the specific application of 3D printing technology in material field and biology field. However, recognizing the change of research hotspot in the field according to vocabulary change only belongs to static explanation. It is still necessary to recognize the development and future trend of 3D printing technology through the documents formed by tracking mutation in citation network. Based on this, the articles with the citation times ranking top 100 in 2010-2014 will be chosen for analysis.

The visualization chart of current mainstream research field and corresponding time axis can be drawn according to the data set. Time axis visualization can highlight the emerging research field. Thus, it plays an important role in the recognition aspect. According to the chart drawn, we can know in recent five years, the theme words of 3D printing field mainly include 3D printing processing technique, tissue, organ and cell. Hence, subject development trend in recent five years should be taken into account to guarantee correct grasp of hot contents in the field. The latest research groups mainly include low temperature storage, role, quality, hydrogel produced by ion covalent bond tangling and thermal performance etc. ${ }^{[5]}$ Thus, these will be researched and analyzed next to gain current research hotspots.

In terms of low temperature storage, there are 24 documents. After analysis of documents in the group, we can find that the research scope is mainly the set of 3D printing equipment, other chemical technologies and biotechnology, like research and development of 3D printing resin material.

In terms of the role, the application of 3D printing technology in biology field and tissue engineering is reflected. In the journal Science, the application of 3D printing technology in tissue engineering is expected. The specific contents involve the decrease of organ transplantation rate and bio-implantation of 3D printing technology. In current stage, many famous biology periodicals pay attention to this field and especially the application development of 3D printing technology in biology field. The research content on the organ is same with role research content. The sole difference is that organ research pays more attention to availability of medical implantation materials manufactured by 3D printing and biocompatibility, i.e. actual quality of 3D printing bio-manufacturing. It thus can be seen that the application of 3D printing technology in bio-manufacturing has a bright prospect. However, the development of this technology is not perfect, and researchers still make efforts.

In terms of hydrogel produced by ion covalent bond tangling and thermal performance, the scale is not large. The research subject is 3D printing material. Compared with the group of thermal performance, hydrogel produced by ion covalent bond tangling pays more attention to 3D bio-printing, mainly including tissue engineering and regenerative medicine. The group of thermal 
performance involves the research on traditional 3D printing material, i.e. the application of metal 3D printing technology. Thus, metal 3D printing technology will complement with manufacturing process to achieve synergetic development in the future development process. This has great development space.

Based on this, it is found from the research on the key word "key challenge" that, the challenge not merely includes material, equipment and technology, but also covers industrial standard setting, business and manufacturing mode reform about 3D printing technology. Meanwhile, the development of 3D printing technology leads to multiple problems such as intellectual property protection and social ethics.

\section{Conclusions}

In conclusion, this paper overall collects and settles paper publishing quantity about 3D printing field at home and aboard. Except the research in the development trend of this field, mainstream academic group, research theme and hot issues are further analyzed. The mainstream academic groups with great influence in 3D printing field are discussed. Printing also covers many subject knowledge systems. In essence, evolution is harmonious development of industry chain. According to citation analysis and research of 3D printing technology field, we can know existing researches pay high attention to the influence of business and manufacturing mode of 3D printing technology. This technology will achieve organic combination with internet field and cloud computing field to achieve total innovation of business and manufacturing mode. In such case, the research on 3D printing technology from the system perspective will be concerned. Meanwhile, a new social reform will be promoted. This paper regards 3D printing technology as the main research object and sets forth the evolution and development frontier of this technology through document analysis, in the hope of offering certain help.

\section{References}

[1] Wang Canyou, Su Qin, Analysis of the Effects of Policy Measures on Emerging Technology Evolution - A Case Study of 3D Printing Technology, Science \& Technology Progress and Policy, 2016,33(6):110-116.

[2] Gao Qun, Zheng Jialin, Mechanism Research of Inter- organizational Learning in Industry Alliance Based on Evolutionary Game - Exemplified by the 3D Printing Technology Industry, Science and Technology Management Research, 2016,36(4):145-151.

[3] Li Dengjie, Zhai Dongsheng, Feng Xiuzhen et al., Recognition and Evolution Analysis of Key-entity Based on Dynamic Network-Using 3D Printing Technology Patent as an Example, Science and Technology Management Research, 2016(8):114-119.

[4] Wang Canyou, Su Qin, Evolution and Development Trends of 3D Printing Technology Based on the Analysis of Literature, Journal of Intelligence, 2015(9):72-77,71.

[5] Yu Huan, Wang Xue, Liu Siyu et al., Analysis of Emerging Technology Research Evolution Based on Mapping Knowledge Domain - A Case Study of Hotspot and Frontier of 3D Printing Technology, Modern Business, 2014(35):277-278. 